\title{
Improving the lower bound to the secret-key capacity of the thermal amplifier channel ${ }^{\star}$
}

\author{
Gan Wang ${ }^{1,2}$, Carlo Ottaviani ${ }^{2}$, Hong Guo ${ }^{1}$, and Stefano Pirandola ${ }^{2,3, a}$ \\ ${ }^{1}$ State Key Laboratory of Advanced Optical Communication Systems and Networks, School of Electronics \\ Engineering and Computer Science, and Center for Quantum Information Technology, Peking University, \\ Beijing 100871, P.R. China \\ 2 Computer Science, University of York, York YO10 5GH, UK \\ 3 Research Lab of Electronics, MIT, Cambridge, MA 02139, USA
}

Received 16 July 2018 / Received in final form 13 November 2018

Published online 17 January 2019

(C) The Author(s) 2019. This article is published with open access at Springerlink.com

\begin{abstract}
We consider the noisy thermal amplifier channel, where signal modes are amplified together with environmental thermal modes. We focus on the secret-key capacity of this channel, which is the maximum amount of secret bits that two remote parties can generate by means of the most general adaptive protocol, assisted by unlimited and two-way classical communication. For this channel only upper and lower bounds are known, and in this work we improve the lower bound. We consider a protocol based on squeezed states and homodyne detections, in both direct and reverse reconciliation. In particular, we assume that trusted thermal noise is mixed on beam splitters controlled by the parties in a way to assist their homodyne detections. The new improved lower bounds to the secret-key capacity are obtained by optimizing the key rates over the variance of the trusted noise injected, and the transmissivity of the parties' beam splitters. Our results confirm that there is a separation between the coherent information of the thermal amplifier channel and its secret key capacity.
\end{abstract}

\section{Introduction}

In the past decades, quantum information science $[1,2]$ has successfully achieved a huge amount of goals. In particular, quantum key distribution (QKD) has emerged as the most mature quantum technology. The aim of QKD is to distribute secret keys between two parties, a sender (Alice) and a receiver (Bob), who perform a communication scheme in two stages. The first stage is quantum communication over a quantum channel controlled by an eavesdropper (Eve), ending with Alice and Bob sharing a raw key. During the second stage of classical communication $(\mathrm{CC})$, the parties run a classical protocol of error correction, sifting and privacy amplification. In this way, they extract a shorter key over which Eve only has a negligible amount of knowledge. The fundamental mechanism ensuring security is the no-cloning theorem [3], which forbids a perfect copy of the non-orthogonal signal states sent by Alice.

Two main designs of QKD exist. One is based on qubits [4], the other is based on continuous-variable (CV) quantum systems $[5,6]$, which are described by

\footnotetext{
* Contribution to the Topical Issue "Quantum Correlations", edited by Marco Genovese, Vahid Karimipour, Sergei Kulik, and Olivier Pfister.

a e-mail: stefano.pirandola@york.ac.uk
}

an infinite-dimensional Hilbert space. In particular, Gaussian CV QKD [6] received a lot of attention for the relative simplicity of its theoretical analysis, and the simplicity of its experimental realization based on cheap, off-the-shelf, linear optical elements and highly efficient homodyne detectors, even at non-standard frequencies [7]. During the past years, the research in Gaussian QKD has led to the design and experimental implementation of a number of protocols, including one-way [8-23], and two-way [24-31] schemes, as well as the study of measurement-device-independent [32-38] schemes.

An important goal in this research area is to determine the optimal secret key rate, or secret-key capacity, over the various models of quantum communication channels. This computation is generally complicated due to the fact that feedback has to be taken into account. More precisely, one has to optimize the key-rate over adaptive LOCCs, i.e., local operations (LOs) assisted by unlimited two-way CC. The combined use of the relative entropy of entanglement (REE) and teleportation stretching allowed PLOB [39] to upperbound the secret-key capacities of Pauli channels, erasure channels, amplitude damping channels, and bosonic Gaussian channels (see also the follow-up works [40-44]). Among the Gaussian channels, the thermal loss channel and the thermal amplifier are the most interesting and important. In a previous work [45], we showed how the lower bound to the secret key capacity 
of the thermal loss channel can be improved by exploiting the benefits of injecting trusted thermal noise $[12,18]$. This type of analysis has not been yet performed for the thermal amplifier channel.

In this work, we improve the lower bound to the secret-key capacity of the thermal amplifier channel by computing the achievable rate of a QKD protocol based on squeezed states and homodyne detections. We assume that the parties possess quantum memory so that they do not need to reconcilate their bases, i.e., the choices of the $q$ or the $p$ quadrature. We also assume that trusted thermal noise is locally used by Alice or Bob, depending on whether the protocol is implemented in direct reconcilation (DR) or reverse reconciliation (RR). Under these conditions, the lower bound based on the coherent information $[46,47]$ is always beaten by the rate in DR (and also outperformed by the $\mathrm{RR}$ rate in a small region for low gains and high thermal noise).

\section{Upper and lower bounds to the secret-key capacity of the thermal amplifier}

Consider two parties, Alice and Bob, performing an adaptive protocol over a quantum channel $\mathcal{E}$. After $n$ uses, they share the output state $\rho_{n}:=\rho\left(\mathcal{E}^{\otimes}\right)$ which depends on the sequence of adaptive LOCCs performed, i.e., $\mathcal{L}=$ $\left\{\Lambda_{0}, \Lambda_{1}, \ldots, \Lambda_{n}\right\}$. Let $\phi_{n}$ be a private target state [48] with information content equal to $n R_{n}$ secret bits. The output state $\rho_{n}$ and $\phi_{n}$ fulfill the $\epsilon$-security relation $\left\|\rho_{n}-\phi_{n}\right\| \leq \epsilon$. Now, the generic two-way capacity of the channel can be obtained by optimizing over all the possible LOCC-sequences $\mathcal{L}$, and by taking the limit of infinite channel uses, i.e., $n \rightarrow \infty$. In formulas, we can define the secret key capacity as follows

$$
\mathcal{K}(\mathcal{E}):=\sup _{\mathcal{L}} \lim _{n \rightarrow \infty} R_{n}
$$

This quantity gives the maximum achievable number of secret bits that can be transmitted per channel use.

Let us introduce the quadrature vector $\hat{\mathbf{x}}:=(q, p)^{T}$. Then, a thermal amplifier channel $\mathcal{E}_{g, \bar{n}}$ corresponds to the transformation

$$
\hat{\mathbf{x}} \rightarrow \sqrt{g} \hat{\mathbf{x}}+\sqrt{g-1} \hat{\mathbf{x}}_{E}
$$

where $g>1$ is the gain, and $\hat{\mathbf{x}}_{E}$ are the quadratures of a thermal environment mode $E$ with $\bar{n}$ mean number of photons. Let us set $\omega=2 \bar{n}+1$ and

$$
h(x):=\frac{x+1}{2} \log _{2} \frac{x+1}{2}-\frac{x-1}{2} \log _{2} \frac{x-1}{2} .
$$

Then, we may write the secret-key capacity of the thermal amplifier channel $\mathcal{K}\left(\mathcal{E}_{g, \bar{n}}\right)$ as

$$
\Omega(g, \bar{n}) \leq \mathcal{K}\left(\mathcal{E}_{g, \bar{n}}\right) \leq \Phi(g, \bar{n}),
$$

where the lower bound [49] is given by

$$
\Omega(g, \bar{n})=\log _{2}\left(\frac{g}{g-1}\right)-h(\omega)
$$

and corresponds to the coherent information of the channel, which is defined as the coherent information of its (asymptotic) Choi matrix [39,50]. In equation (4), the upper bound is computed from the REE [51-53] of the (asymptotic) Choi matrix and is equal to [39]

$$
\Phi(g, \bar{n})=\left\{\begin{array}{l}
\log _{2}\left(\frac{g^{\bar{n}+1}}{g-1}\right)-h(\omega), \text { for } \bar{n}<(g-1)^{-1} \\
0, \text { otherwise. }
\end{array}\right.
$$

\section{Improving the lower bound}

We now present a QKD protocol whose key rate in DR and RR improves the lower bound in equation (4). Even though the improvement found is small, it is meaningful because it shows that the coherent information of the thermal amplifier channel cannot be its secret key capacity. For instance, when Eve has $\bar{n}=1$ photon, the key rate drops to 0 when transmissivity is approximately 1.33 for the previously known lower bound in equation (5), while drops to 0 when transmissivity is 1.38 for the improved lower bound. First we derive the new achievable rates in Sections 3.1 and 3.2. Then we numerically compare the results in Section 3.3.

\subsection{Achievable rate in direct reconciliation}

We show the following result.

Theorem 1. Consider a thermal amplifier channel, with gain $g$ and thermal noise $\omega$. Its secret key rate is lowerbounded by the achievable DR rate

$$
R \bullet(g, \omega)=\max _{\eta_{A}, \gamma} R^{\triangleright}\left(g, \omega, \eta_{A}, \gamma\right),
$$

where

$$
\begin{aligned}
R^{\triangleright}\left(g, \omega, \eta_{A}, \gamma\right):= & \frac{1}{2} \log _{2} \frac{g\left[g \eta_{A} \omega+\gamma(g-1)\left(1-\eta_{A}\right)\right]}{(g-1)\left[g \gamma\left(1-\eta_{A}\right)+\eta_{A} \omega(g-1)\right]} \\
& +h\left(\sqrt{\frac{\omega\left[g \eta_{A}+\gamma \omega(g-1)\left(1-\eta_{A}\right)\right]}{g \eta_{A} \omega+\gamma(g-1)\left(1-\eta_{A}\right)}}\right) \\
& -h(\omega)
\end{aligned}
$$

and the maximization is over transmissivity $\eta_{A}$ of a beam splitter at Alice's side, and the thermal variance $\gamma \geq 1$.

Proof. Consider the Gaussian CV-QKD protocol described in Figure 1. We study its security in the entanglement-based (EB) representation. Thus, we assume that Alice has a two-mode squeezed vacuum (TMSV) state $\Phi^{\mu}$ of modes $A_{0}$ and $B_{0}$. The covariance 
matrix $(\mathrm{CM})$ describing this zero mean Gaussian state is the following [6]

$$
\mathbf{V}_{A_{0} B_{0}}=\mathbf{V}_{\mathrm{TMSV}}(\mu):=\left(\begin{array}{cc}
\mu \mathbf{I} & \sqrt{\mu^{2}-1} \mathbf{Z} \\
\sqrt{\mu^{2}-1} \mathbf{Z} & \mu \mathbf{I}
\end{array}\right),
$$

where $\mathbf{I}=\operatorname{diag}(1,1)$ and $\mathbf{Z}=\operatorname{diag}(1,-1)$, and $\mu$ is the variance of the TMSV state. Alice's local mode $A_{0}$ is processed by a beam splitter with transmissivity $\eta_{A}$, together with mode $v$ in a thermal state of variance $\gamma$, and $\mathrm{CM} \mathbf{V}_{v}=\gamma \mathbf{I}$. One of the outputs, $A^{\prime}$, is discarded, while the other, $A$, is homodyned randomly switching between quadrature $q$ and $p$. This operation prepares thermal states in the travelling mode $B_{0}$.

Mode $B_{0}$ is sent through the thermal amplifier channel with gain $g>1$ and thermal noise $\omega=2 \bar{n}+1$. The thermal input mode $E$ is part of Eve's TMSV state with $\mathrm{CM} \mathbf{V}_{e E}=\mathbf{V}_{\mathrm{TMSV}}(\omega)$ as in Figure 1. Eve's output modes $e$ and $E^{\prime}$ are stored in a quantum memory, which is coherently measured at the end of the protocol (collective attack). The channel output $B$, travelling to Bob, is stored in Bob's quantum memory (BQM) for later measurements. After many uses of the channel $(n \gg 1)$, Alice communicates which quadrature she has measured in each round, thus Bob can perform exactly the same sequence of homodyne detections on the stored modes, and then infer the outcomes of Alice's preparation stage.

The initial global state $\rho_{0}$ of Alice, Bob and Eve is given by the tensor product $\rho_{0}=\rho_{A_{0} B_{0}} \otimes \rho_{e E} \otimes \rho_{v}$, having CM $\mathbf{V}_{0}^{\bullet}=\mathbf{V}_{A_{0} B_{0}} \oplus \mathbf{V}_{e E} \oplus \mathbf{V}_{v}=\mathbf{V}_{A_{0} B_{0} e E v}$. For convenience, we rearrange the state as $\mathbf{V}_{0}^{\bullet}=\mathbf{V}_{A_{0} v B_{0} E e}$, and process it by a sequence of symplectic transformation describing the evolution throughout the beam splitter $\left(\eta_{A}\right)$ and the amplifier $(g)$. We first process mode $A_{0}$ and $v$, by applying the symplectic transformation $\tilde{\mathbf{V}}^{\downarrow}=\mathbf{S}_{\eta_{A}} \mathbf{V}_{0} \mathbf{S}_{\eta_{A}}^{T}$, where $\mathbf{S}_{\eta_{A}}:=\mathbf{T}_{B S}\left(\eta_{A}\right) \oplus \mathbf{I} \oplus \mathbf{I} \oplus \mathbf{I}$, with

$$
\mathbf{T}_{B S}\left(\eta_{A}\right):=\left(\begin{array}{cc}
\sqrt{\eta_{A}} \mathbf{I} & \sqrt{1-\eta_{A}} \mathbf{I} \\
-\sqrt{1-\eta_{A}} \mathbf{I} & \sqrt{\eta_{A}} \mathbf{I}
\end{array}\right) .
$$

Then, we process the CM $\tilde{\mathbf{V}}$ to consider the evolution of the state through the thermal amplifier, by applying the symplectic transformation $\mathbf{S}_{g} \tilde{\mathbf{V}} \bullet \mathbf{S}_{g}^{T}$, where $\mathbf{S}_{g}:=$ $\mathbf{I} \oplus \mathbf{I} \oplus \mathbf{T}_{A M P}(g) \oplus \mathbf{I}$, and

$$
\mathbf{T}_{A M P}(g):=\left(\begin{array}{cc}
\sqrt{g} \mathbf{I} & \sqrt{g-1} \mathbf{Z} \\
\sqrt{g-1} \mathbf{Z} & \sqrt{g} \mathbf{I}
\end{array}\right) .
$$

Thus, we can compute the CM V $\mathbf{v}$, corresponding to the quantum state $\rho_{A A^{\prime} B E^{\prime} \text { e }}$ Then we can trace out mode $A^{\prime}$ to obtain the output state $\rho_{A B E^{\prime} e}=\operatorname{Tr}_{A^{\prime}}\left(\rho_{A A^{\prime} B E^{\prime} e}\right)$ with $\mathrm{CM} \mathbf{V}_{A B E^{\prime} e}$. From this $\mathrm{CM}$, we may compute Alice's and Bob's mutual information $I_{A B}$ as well as Eve's Holevo function $\chi_{A E}$, bounding Eve's knowledge on Alice's encoding variables.

Under ideal conditions of perfect reconciliation efficiency, the key rate in DR is given by $R \bullet:=I_{A B}-\chi_{A E}$. We can derive the analytical expression of the asymptotic key rate, when the Gaussian modulation is large $\mu \rightarrow \infty$.

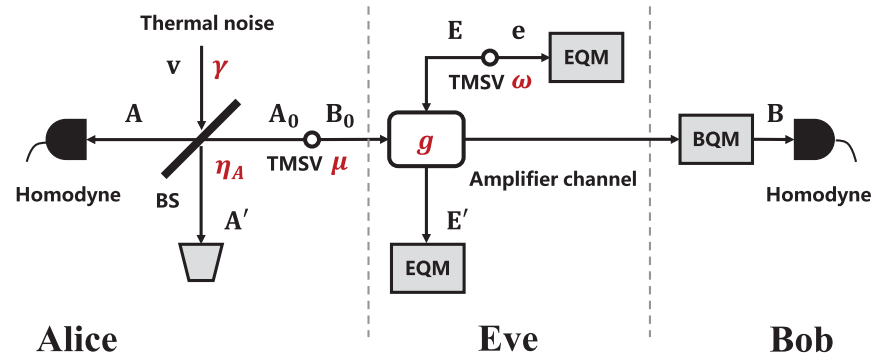

Fig. 1. Protocol with trusted thermal noise in DR. Alice has a TMSV state, whose mode $B_{0}$ is sent to Bob through a thermal amplifier channel with gain $g$. Mode $A_{0}$ is processed by a beam splitter (BS) with transmissivity $\eta_{A}$ together with a thermal mode $v$ with variance $\gamma$, and then measured by a homodyne detector in $q$ or $p$. The attack is performed by Eve, who exploits modes $e$ and $E$ in a TMSV state (with variance $\omega$ ) and stores the output in Eve's quantum memory (EQM). The signals from Alice are stored by Bob in his quantum memory (BQM) and homodyned after Alice has sent to Bob the correct sequence of homodyne detections to perform.

To compute $I_{A B}$, let us first consider the CM describing modes $A$ and $B$. This is given by the following expression

$$
\mathbf{V}_{A B}=\left(\begin{array}{cc}
{\left[\eta_{A} \mu+\left(1-\eta_{A}\right) \gamma\right] \mathbf{I}} & \sqrt{g \eta_{A}\left(\mu^{2}-1\right)} \mathbf{Z} \\
\sqrt{g \eta_{A}\left(\mu^{2}-1\right) \mathbf{Z}} & {[g \mu+(g-1) \omega] \mathbf{I}}
\end{array}\right),
$$

from which we can extract Alice's variance $V_{A}=\eta_{A} \mu+$ $\left(1-\eta_{A}\right) \gamma$. Applying homodyne detection on mode $B$ we obtain the following expression for Alice's variance conditioned to Bob outcomes

$$
V_{A \mid \beta}=\frac{g \gamma\left(1-\eta_{A}\right)+\eta_{A} \omega(g-1)}{g} .
$$

From the expression of $V_{A}$ and $V_{A \mid \beta}$, and using the definition of mutual information $I_{A B}=\frac{1}{2} \log _{2} V_{A} V_{A \mid \beta}^{-1}$, we obtain the asymptotic Alice and Bob's mutual information, which is given by

$$
I_{A B} \stackrel{\mu \rightarrow \infty}{=} \frac{1}{2} \log _{2} \frac{g \eta_{A} \mu}{g \gamma\left(1-\eta_{A}\right)+\eta_{A} \omega(g-1)} .
$$

We then compute Eve's Holevo function, defined as $\chi_{A E}:=S_{T}-S_{C}$, where $S_{T}$ is the von Neumann entropy of $\rho_{E^{\prime} e}$, and $S_{C}$ is that of the conditional state $\rho_{E^{\prime} e \mid A}$. We consider the block of $\mathrm{CM} \mathbf{V}$ given by

$$
\mathbf{V}_{E^{\prime} e A}^{\triangleright}=\left(\begin{array}{cc}
\mathbf{V}_{E^{\prime} e}^{\nabla} & \mathbf{C} \\
\mathbf{C} & \mathbf{V}_{A}
\end{array}\right)
$$

where $\mathbf{V}_{A}=\left[\eta_{A} \mu+\left(1-\eta_{A}\right) \gamma\right] \mathbf{I}$, and

$$
\begin{aligned}
\mathbf{V}_{E^{\prime} e} & =\left(\begin{array}{c}
{[(g-1) \mu+g \omega] \mathbf{I} \sqrt{g\left(\omega^{2}-1\right)} \mathbf{Z}} \\
\sqrt{g\left(\omega^{2}-1\right)} \mathbf{Z} \quad \omega \mathbf{I}
\end{array}\right), \\
\mathbf{C} & =\left(\begin{array}{c}
\sqrt{(g-1) \eta_{A}\left(\mu^{2}-1\right)} \mathbf{I} \\
0 \mathbf{I}
\end{array}\right) .
\end{aligned}
$$


We then compute the asymptotic symplectic spectrum of $\mathbf{V}_{E^{\prime} e}$, obtaining the following symplectic eigenvalues

$$
\left\{\nu_{1}, \nu_{2}\right\} \stackrel{\mu \rightarrow \infty}{\rightarrow}\{(g-1) \mu, \omega\} .
$$

The total von Neumann entropy is $S_{T}=h\left(\nu_{1}\right)+h\left(\nu_{2}\right)$. Considering that $h(x)=\log _{2}(e x / 2)$ for $x \rightarrow \infty$, we can obtain the following asymptotic formula

$$
S_{T} \stackrel{\mu \rightarrow \infty}{=} \log _{2} \frac{e}{2}(g-1) \mu+h(\omega) .
$$

After Alice's homodyne detection of quadrature $q$ (or $p$ ) on mode $A$, we also obtain Eve's conditional CM

$$
\mathbf{V}_{E^{\prime} e \mid A}^{\triangleright}=\mathbf{V}_{E^{\prime} e}-\mathbf{C}\left(\Pi \mathbf{V}_{A} \Pi\right)^{-1} \mathbf{C}^{T}
$$

where $\Pi=\operatorname{diag}(1,0)$ for homodyne detection on $q$ and $\Pi=\operatorname{diag}(0,1)$ for homodyne detection on $p$. From equation (19) we can compute the symplectic spectrum of $\mathbf{V}_{E^{\prime} e \mid A}$. After some algebra and working in the limit of large modulation $(\mu \rightarrow \infty)$, we obtain the analytical expressions of the symplectic eigenvalues

$$
\begin{aligned}
& \bar{\nu}_{1} \stackrel{\mu \rightarrow \infty}{=} \sqrt{\frac{(g-1)\left[g \eta_{A} \omega+\gamma(g-1)\left(1-\eta_{A}\right)\right]}{\eta_{A}} \mu,} \\
& \bar{\nu}_{2} \stackrel{\mu \rightarrow \infty}{=} \sqrt{\frac{\omega\left[g \eta_{A}+\gamma \omega(g-1)\left(1-\eta_{A}\right)\right]}{g \eta_{A} \omega+\gamma(g-1)\left(1-\eta_{A}\right)}} .
\end{aligned}
$$

From this symplectic spectrum we can compute the conditional von Neumann entropy $S_{C}=h\left(\bar{\nu}_{1}\right)+h\left(\bar{\nu}_{2}\right)$. For large $\mu$, it becomes

$$
\begin{aligned}
S_{C} \stackrel{\mu \rightarrow \infty}{=} & \frac{1}{2} \log _{2} \frac{e^{2}}{4} \frac{(g-1)\left[g \eta_{A} \omega+\gamma(g-1)\left(1-\eta_{A}\right)\right]}{\eta_{A}} \mu \\
& +h\left(\bar{\nu}_{2}\right) .
\end{aligned}
$$

Combining equations (18) and (21) in the definition of the Holevo function $\chi_{A E}:=S_{T}-S_{C}^{\bullet}$, we derive

$\chi_{A E} \stackrel{\mu \rightarrow \infty}{=} \frac{1}{2} \log _{2} \frac{(g-1) \eta_{A} \mu}{g \eta_{A} \omega+\gamma(g-1)\left(1-\eta_{A}\right)}+h(\omega)-h\left(\vec{\nu}_{2}\right)$.

Finally, using equations (14) and (22), we obtain the analytic expression of the asymptotic key rate in DR, which is given in equation (8).

The secret key rate of equation (8) can be optimized over Alice's free parameters, which are the transmissivity $\eta_{A} \in[0,1]$ and the variance $\gamma \geq 1$. When $\eta_{A}=1$, which means we have no trusted noise injected by Alice, it is easy to verify that $R \triangleright(g, \omega, 1, \gamma)=\log _{2}[g /(g-1)]-h(\omega)$, corresponding to the previous lower bound $\Omega$ in equation (5). It is therefore clear that the optimized achievable rate $R$ in equation (7) is $\geq \Omega$ for any value of the gain. In the numerical comparison below (Sec. 3.3) we explicitly show that there is a strict separation, so that we have $R \nabla>\Omega$ in a wide range.

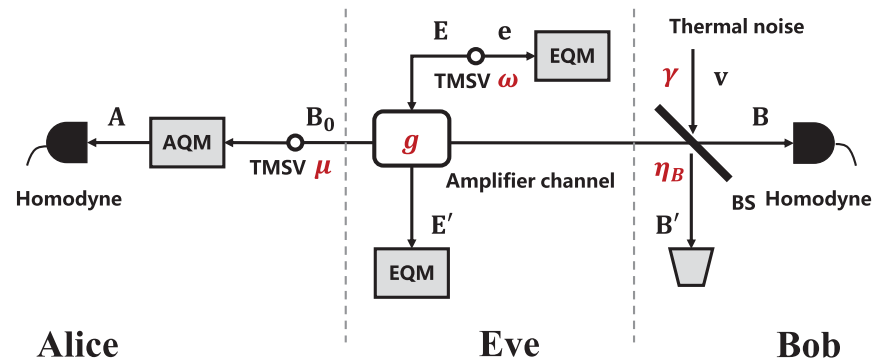

Fig. 2. Protocol with trusted thermal noise in RR. Alice starts from a TMSV where mode $B_{0}$ is sent to Bob through a thermal amplifier channel with gain $g$ (and thermal noise $\omega$ ), while mode $A_{0}$ is stored in Alice's quantum memory (AQM), waiting for the correct sequence of homodyne detections, which is announced by Bob at the end of the protocol. The attack is assumed to be collective, with Eve using a TMSV state, whose output modes, $e$ and $E^{\prime}$, are stored in her quantum memory (EQM). At the channel output, the signals are processed within Bob's private space, by a beam splitter (BS) with transmissivity $\eta_{B}$ and a thermal mode $v$ with variance $\gamma$. The signal modes are then homodynes either in $q$ or $p$. At the end, Bob publicly declares to Alice his sequence of homodynes. At this point, Alice performs the correct sequence of homodyne detections on the modes $A$ she stored in her quantum memory.

\subsection{Achievable rate in reverse reconciliation}

We now show the following.

Theorem 2. Consider a thermal amplifier channel with gain $g$ and thermal noise $\omega$. Its secret key rate is lowerbounded by the achievable $R R$ rate

$$
R^{\triangleleft}(g, \omega)=\max _{\eta_{B}, \gamma} R^{\triangleleft}\left(g, \omega, \eta_{B}, \gamma\right)
$$

where

$$
\begin{aligned}
R^{\triangleleft}\left(g, \omega, \eta_{B}, \gamma\right):= & \frac{1}{2} \log _{2} \frac{\eta_{B} \omega+\gamma(g-1)\left(1-\eta_{B}\right)}{(g-1)\left[\gamma\left(1-\eta_{B}\right)+\eta_{B} \omega(g-1)\right]} \\
& +h\left(\sqrt{\frac{\omega\left[\eta_{B}-\gamma \omega(g-1)\left(1-\eta_{B}\right)\right]}{\eta_{B} \omega+\gamma(g-1)\left(1-\eta_{B}\right)}}\right) \\
& -h(\omega),
\end{aligned}
$$

and the maximization is over the transmissivity $\eta_{B}$ of Bob's beam splitter, and the thermal variance $\gamma \geq 1$.

Proof. The proof is similar to the DR discussed in previous section. Consider the Gaussian protocol in Figure 2. Alice starts from the same TMSV state $\Phi^{\mu}$, of modes $A_{0}$ and $B_{0}$, given in equation (9). Now, it is Alice's mode $A$ that is stored in Alice's quantum memory (AQM) for later measurements, while mode $B_{0}$ travels to Bob through the amplifier channel. Bob implements a noisy detection, mixing the input mode with a thermal mode $v$ with variance $\gamma$ via a beam splitter whose transmissivity is $\eta_{B}$. Then Bob measures the $q$ or the $p$ quadrature (communicating his choices at the end of the quantum communication after $n \gg 1$ rounds).

The initial global state of Alice, Bob and Eve has $\mathrm{CM} \mathbf{V}_{0}^{\boldsymbol{\triangleleft}}=\mathbf{V}_{A B_{0}} \oplus \mathbf{V}_{e E} \oplus \mathbf{V}_{v}=\mathbf{V}_{A_{0} B_{0} e E v}$, and we again 

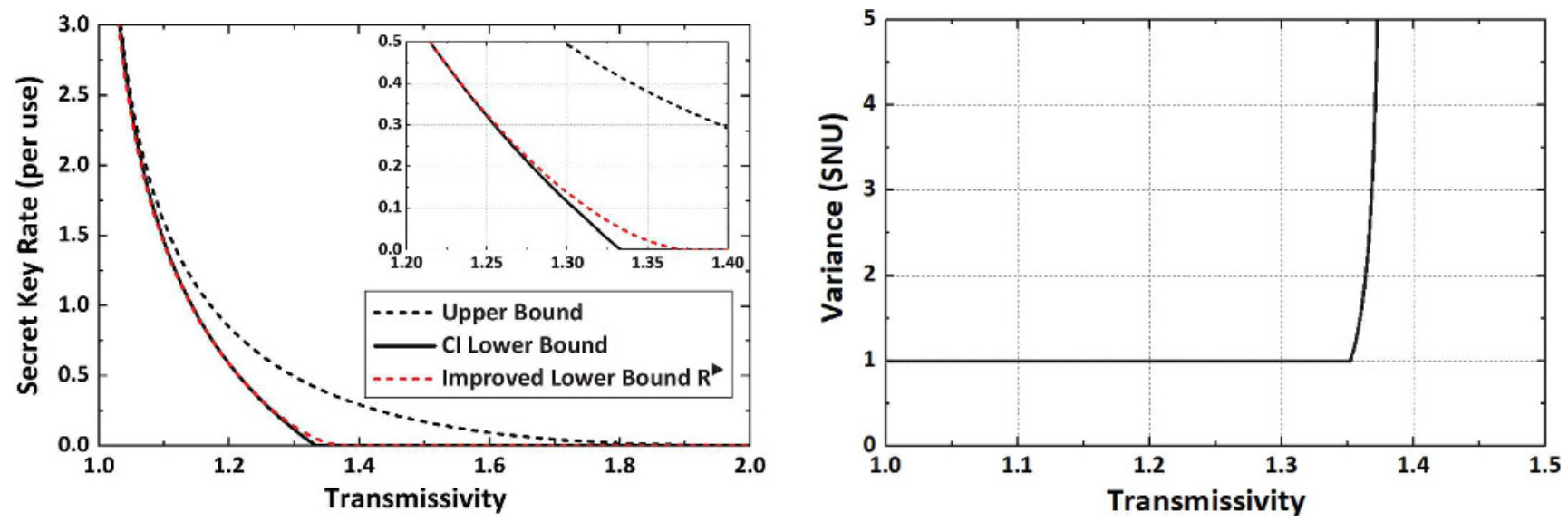

Fig. 3. Comparison between the previous lower bound and the new improved results. In the left panel, we consider a thermal amplifier channel with $\bar{n}=1$ photons and arbitrary gain $g$. We then compare the new lower bound $R^{\triangleright}$ of equation (7) (reddashed line) with the coherent information of the channel $\Omega$ of equation (5) (solid-black line). We also show the upper bound $\Phi$ of equation (6) (black-dashed line). In the right panel, we show the optimal variance of the trusted noise.

rearrange the modes so that $\mathbf{V}_{0}^{\mathbf{U}}=\mathbf{V}_{A_{0} v B_{0} E e}$. This state is processed by the amplifier $(g)$ and then the beam splitter $\left(\eta_{B}\right)$. First we obtain $\tilde{\mathbf{V}}^{\triangleleft}=\mathbf{S}_{g} \mathbf{V}_{0}^{\boldsymbol{4}} \mathbf{S}_{g}^{T}$, where $\mathbf{S}_{g}$ has been defined above, and then we compute $\mathbf{V}^{\mathbf{4}}=$ $\mathbf{S}_{\eta_{B}} \tilde{\mathbf{V}} \triangleleft \mathbf{S}_{\eta_{B}}^{T}$, where $\mathbf{S}_{\eta_{B}}:=\mathbf{I} \oplus \mathbf{T}_{B S}\left(\eta_{B}\right) \oplus \mathbf{I} \oplus \mathbf{I}$, with $\mathbf{T}_{B S}(\cdot)$ and $\mathbf{T}_{A M P}(\cdot)$ given in equations (10) and (11). Discarding Bob's mode $B^{\prime}$, we compute the output state $\rho_{A B E^{\prime} e}=\operatorname{Tr}_{B^{\prime}}\left(\rho_{A B^{\prime} B E^{\prime} e}\right)$ with CM $\mathbf{V}_{A B E^{\prime} e}$. From this $\mathrm{CM}$, we may compute the secret key rate in $\mathrm{RR}$, i.e.,

$$
R^{\triangleleft}=I_{A B}-\chi_{B E},
$$

where $\chi_{B E}$ is Eve's Holevo information on Bob's outcomes. The analytical form of $R^{\boldsymbol{4}}$ is obtained under the assumption of large modulation $\mu \rightarrow \infty$.

The CM describing Alice's and Bob's output modes $A$ and $B$ is the following

$$
\mathbf{V}_{A B}^{\mathbf{A}}=\left(\begin{array}{cc}
\mu \mathbf{I} & \sqrt{g \eta_{B}\left(\mu^{2}-1\right)} \mathbf{Z} \\
\sqrt{g \eta_{B}\left(\mu^{2}-1\right)} \mathbf{Z} & {\left[\eta_{B}(g \mu+(g-1) \omega)+\left(1-\eta_{B}\right) \gamma\right] \mathbf{I}}
\end{array}\right) .
$$

We can therefore compute Alice and Bob's mutual information

$$
I_{A B} \stackrel{\mu \rightarrow \infty}{=} \frac{1}{2} \log _{2} \frac{g \eta_{B} \mu}{\gamma\left(1-\eta_{B}\right)+(g-1) \eta_{B} \omega} .
$$

Eve's Holevo information can be written as $\chi_{B E}=S_{T}^{\boldsymbol{4}}-$ $S_{C}^{\boldsymbol{\triangleleft}}$, where $S_{T}^{\boldsymbol{\triangleleft}}$ is the von Neumann entropy for Eve's total state $\rho_{E^{\prime} e}$, while $S_{C}$ is obtained from the conditional quantum state $\rho_{E^{\prime} e \mid B}$. For its computation, consider the following CMs

$$
\begin{aligned}
& \mathbf{V}_{B E^{\prime} e}^{\boldsymbol{\Delta}}=\left(\begin{array}{cc}
\mathbf{V}_{E^{\prime} e}^{\boldsymbol{4}} & \overline{\mathbf{C}} \\
\mathbf{C}_{B} & \mathbf{V}_{B}
\end{array}\right), \\
& \mathbf{V}_{E^{\prime} e}^{\mathbf{A}_{e}}=\left(\begin{array}{cc}
{[(g-1) \mu+g \omega] \mathbf{I}} & \sqrt{g\left(\omega^{2}-1\right)} \mathbf{Z} \\
\sqrt{g\left(\omega^{2}-1\right)} \mathbf{Z} & \omega \mathbf{I}
\end{array}\right),
\end{aligned}
$$

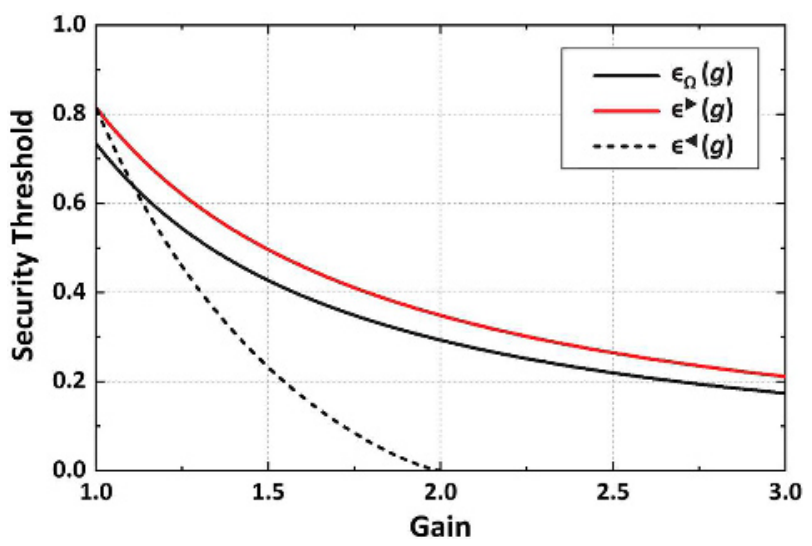

Fig. 4. Comparison of the security threshold $\epsilon_{\Omega}(g)$ (black-solid line), $\epsilon^{\bullet}(g)$ (red-solid line) and $\epsilon^{\boldsymbol{\top}}(g)$ (black-dashed line).

$$
\overline{\mathbf{C}}=\left(\begin{array}{l}
\sqrt{g(g-1) \eta_{B}}(\mu+\omega) \mathbf{Z} \\
\sqrt{(g-1) \eta_{B}\left(\omega^{2}-1\right) \mathbf{I}}
\end{array}\right)
$$

where $\mathbf{V}_{B}=\left[\eta_{B}(g \mu+(g-1) \omega)+\left(1-\eta_{B}\right) \gamma\right]$ I. Clearly, we need to compute only Eve's conditional symplectic spectrum, obtained from Eve and Bob's CM, V $\overrightarrow{B E}^{\prime}$ e by applying homodyne detection on Bob mode $B$. This provides the conditional $\mathrm{CM}$

$$
\mathbf{V}_{E^{\prime} e \mid B}^{\boldsymbol{\triangleleft}}=\mathbf{V}_{E^{\prime} e}^{\boldsymbol{\triangleleft}}-\overline{\mathbf{C}}\left(\Pi \mathbf{V}_{B} \Pi\right)^{-1} \overline{\mathbf{C}}^{T},
$$

whose symplectic eigenvalues have the following asymptotic expressions

$$
\begin{aligned}
& \bar{\nu}_{1}^{\triangleleft} \stackrel{\mu \rightarrow \infty}{=} \sqrt{\frac{(g-1)\left[\eta_{B} \omega+\gamma(g-1)\left(1-\eta_{B}\right)\right]}{g \eta_{B}}} \mu, \\
& \bar{\nu}_{2}^{\triangleleft} \stackrel{\mu \rightarrow \infty}{=} \sqrt{\frac{\omega\left[\eta_{B}+\gamma \omega(g-1)\left(1-\eta_{B}\right)\right]}{\eta_{B} \omega+\gamma(g-1)\left(1-\eta_{B}\right)}} .
\end{aligned}
$$


Therefore, the asymptotic Eve's Holevo information is given by

$\chi_{B E} \stackrel{\mu \rightarrow \infty}{=} \frac{1}{2} \log _{2} \frac{(g-1) \mu}{\eta_{B} \omega+\gamma(g-1)\left(1-\eta_{B}\right)}+h(\omega)-h\left(\bar{\nu}_{2}\right)$.

Combining equations (27) and (31), in equation (25), we find the formula of the asymptotic key rate in $R R$ in the asymptotic limit of large Gaussian modulation, which coincides with that given in equation (24). The secret key rate of equation (24) is then optimized over Bob's free parameters $\eta_{B} \in[0,1]$ and $\gamma \geq 1$.

\subsection{Comparison}

The performances of the new lower bounds are summarized in Figure 3. The left panel compares the improved lower bound in the DR $R$ of equation (7) (red-dashed line) with respect to the previous lower bound $\Omega$ of equation (5) given by the coherent information of the channel (black-solid line). We also show the upper bound $\Phi$ of equation (6) denoted by the black-dashed line. Then, the right panel of Figure 3 shows the optimal variance of the trusted noise used in the improved lower bound $R$.

Further, we compare the security thresholds in Figure 4. Let us define the excess noise of the thermal amplifier channel as $\epsilon=(g-1)(\omega-1) / g$. Then, we may write the rates as $R=R(g, \epsilon)$. Setting $R=0$, we therefore find the maximally tolerable excess noise as a function of the gain, i.e., $\epsilon=\epsilon(g)$. Starting from $\Omega$ and the two optimized rates $R$ and $R^{\triangleleft}$, we therefore compute the corresponding security thresholds $\epsilon_{\Omega}(g), \epsilon^{\star}(g)$ and $\epsilon^{\boldsymbol{}}(g)$ which are plotted in the right panel of Figure 3. As we can see, $\epsilon>(g)>\epsilon_{\Omega}(g)$ for any $g$, while $\epsilon^{\boldsymbol{\top}}(g)$ outperforms $\epsilon_{\Omega}(g)$ only for small gains.

\section{Conclusions}

In this work, we have studied a QKD protocol whose rate is able to improve the lower bound to the secretkey capacity of the thermal amplifier channel. In DR this improvement occurs for any value of the gain $g$ and the thermal noise $\bar{n}$ of the channel. Our protocol is based on randomly switched squeezed states and homodyne detections, which are perfectly reconciliated by resorting to a quantum memory. Most importantly, we employ a beam-splitter and trusted thermal noise just before the homodyne detector. The large-modulation $(\mu \rightarrow \infty)$ and asymptotic $(n \rightarrow \infty)$ secret key rate is then optimized over the free parameters of the over-all noisy detection. Even though the gap between the new lower bounds and the upper bound is still quite large, our work confirms the fact that the coherent information of the thermal amplifier channel is well separated from its secret key capacity. This also seems to suggest that the distribution of secret keys over this quantum channel might occur at higher rates than the distribution of entanglement or the transmission of quantum information.
C.O. and S.P. acknowledge support from the EPSRC via the 'Quantum Communications HUB' (EP/M013472/1) and the European Union via Continuous Variable Quantum Communications (CiViQ, Project ID: 820466). G.W. and H.G. acknowledge support from the National Natural Science Foundation of China (Grant No. 61531003).

\section{Author contribution statement}

G.W. derived the main results and wrote most of the manuscript. C.O. contributed to supervise the project and edited the manuscript. H.G. contributed to preparation and revision of the manuscript. S.P. set the general problem and edited the manuscript.

Open Access This is an open access article distributed under the terms of the Creative Commons Attribution License (http://creativecommons.org/licenses/by/4.0), which permits unrestricted use, distribution, and reproduction in any medium, provided the original work is properly cited.

\section{References}

1. J. Watrous, The Theory of Quantum Information (Cambridge University Press, Cambridge, 2018)

2. M. Hayashi, Quantum Information Theory: Mathematical Foundation (Springer-Verlag, Berlin, Heidelberg, 2017)

3. W.K. Wootters, W.H Zurek, Nature 299, 802 (1982)

4. V. Scarani et al., Rev. Mod. Phys. 81, 1301 (2009)

5. S.L. Braunstein, P. van Loock, Rev. Mod. Phys. 77, 513 (2005)

6. C. Weedbrook et al., Rev. Mod. Phys. 84, 621 (2012)

7. T. Serikawa, A. Furusawa, arXiv: 1803.06462 (2018)

8. F. Grosshans et al., Nature 421, 238 (2003)

9. C. Weedbrook et al., Phys. Rev. Lett. 93, 170504 (2004)

10. A.M. Lance et al., Phys. Rev. Lett. 95, 180503 (2005)

11. C. Silberhorn, T.C. Ralph, N. Lütkenhaus, G. Leuchs, Phys. Rev. Lett. 89, 167901 (2002)

12. R. García-Patrón, N.J. Cerf, Phys. Rev. Lett. 102, 130501 (2009)

13. R. Filip, Phys. Rev. A 77, 022310 (2008)

14. V.C. Usenko, R. Filip, Phys. Rev. A 81, 022318 (2010)

15. C. Weedbrook, S. Pirandola, T.C. Ralph, Phys. Rev. Lett. 105, 110501 (2010)

16. C. Weedbrook, S. Pirandola, S. Lloyd, T.C. Ralph, Phys. Rev. A 86, 022318 (2012)

17. C.S. Jacobsen, T. Gehring, U.L. Andersen, Entropy 17, $4654(2015)$

18. V.C. Usenko, R. Filip, Entropy 18, 20 (2016)

19. V.C. Usenko, F. Grosshans, Phys. Rev. A 92, 062337 (2015)

20. A. Leverrier, F. Grosshans, P. Grangier, Phys. Rev. A 81, $062343(2010)$

21. A. Leverrier, Phys. Rev. Lett. 114, 070501 (2015)

22. F. Furrer et al., Phys. Rev. Lett. 109, 100502 (2012)

23. F. Furrer et al., Phys. Rev. Lett. 112, 019902(E) (2014)

24. S. Pirandola, S. Mancini, S. Lloyd, S.L. Braunstein, Nat. Phys. 4, 726 (2008)

25. C. Ottaviani, S. Mancini, S. Pirandola, Phys. Rev. A 92, 062323 (2015) 
26. C. Ottaviani, S. Pirandola, Sci. Rep. 6, 22225 (2016)

27. C. Weedbrook, C. Ottaviani, S. Pirandola, Phys. Rev. A 89, 012309 (2014)

28. J.H. Shapiro, Phys. Rev. A 80, 022320 (2009)

29. Q. Zhuang, Z. Zhang, J. Dove, F.N.C. Wong, J.H. Shapiro, Phys. Rev. A 94, 012322 (2016)

30. Q. Zhuang, Z. Zhang, N. Lütkenhaus, J.H. Shapiro, Phys. Rev. A 98, 032332 (2018)

31. S. Ghorai, E. Diamanti, A. Leverrier, Composable security of two-way continuous-variable quantum key distribution, arXiv:1806.11356 (2018)

32. S. Pirandola et al., Nat. Photon. 9, 397 (2015)

33. C. Ottaviani, G. Spedalieri, S.L. Braunstein, S. Pirandola, Phys. Rev. A 91, 022320 (2015)

34. Z. Li et al., Phys. Rev. A 89, 052301 (2014)

35. Y. Zhang et al., Phys. Rev. A 90, 052325 (2014)

36. P. Papanastasiou, C. Ottaviani, S. Pirandola, Phys. Rev. A 96, 042332 (2017)

37. C. Lupo, C. Ottaviani, P. Papanastasiou, S. Pirandola, Phys. Rev. A 97, 052327 (2018)

38. C. Lupo, C. Ottaviani, P. Papanastasiou, S. Pirandola, Phys. Rev. Lett. 120, 220505 (2018)

39. S. Pirandola, R. Laurenza, C. Ottaviani, L. Banchi, Nat. Commun. 8, 15043 (2017)
40. T.P.W. Cope, L. Hetzel, L. Banchi, S. Pirandola, Phys. Rev. A 96, 022323 (2017)

41. S. Pirandola, R. Laurenza, L. Banchi, Ann. Phys. 400, 289 (2019)

42. T.P.W. Cope, K. Goodenough, S. Pirandola, J. Phys. A: Math. Theor. 51, 494001 (2018)

43. S. Pirandola, S.L. Braunstein, R. Laurenza, C. Ottaviani, T.P.W. Cope, G. Spedalieri, L. Banchi, Quantum Sci. Technol. 3, 035009 (2018)

44. S. Pirandola, R. Laurenza, S.L. Braunstein, Eur. Phys. J. D 72, $162(2018)$

45. C. Ottaviani et al., Quantum Inf. Sci. Technol. II 9996, 999609 (2016)

46. B. Schumacher, M.A. Nielsen, Phys. Rev. A 54, 2629 (1996)

47. S. Lloyd, Phys. Rev. A 55, 1613 (1997)

48. K. Horodecki, M. Horodecki, P. Horodecki, J. Oppenheim, Phys. Rev. Lett. 94, 160502 (2005)

49. A.S. Holevo, R.F. Werner, Phys. Rev. A 63, 032312 (2001)

50. S. Pirandola, R. García-Patrón, S.L. Braunstein, S. Lloyd, Phys. Rev. Lett. 102, 050503 (2009)

51. V. Vedral, Rev. Mod. Phys. 74, 197 (2002)

52. V. Vedral, M.B. Plenio, M.A. Rippin, P.L. Knight, Phys. Rev. Lett. 78, 2275 (1997)

53. V. Vedral, M.B. Plenio, Phys. Rev. A 57, 1619 (1998) 